\title{
Estimación de la capacidad torácica a través de los huesos
}

Eugenio Aspillaga ${ }^{1}$, Mario GonZÁlez ${ }^{1}$ Y Claudio PAREDES ${ }^{1}$

\section{Introducción}

Conocer la capacidad torácica en forma absoluta o relativa, es un antecedente de evidente utilidad para el estudio de la adaptación de poblaciones humanas a la altura. Sin embargo, esto no ha sido medido de alguna forma en poblaciones humanas extintas, lo que limita el estudio de éstas y el conocimiento del fenómeno de adaptación a la altura en el tiempo.

No hemos encontrado en la literatura una forma de estimar, de una manera absoluta o relativa, la capacidad torácica de un individuo a través de sus huesos, aunque sea en una forma aproximada. Tampoco profesionales del campo de la medicina, anatomía y de la antropología física nos han dado una respuesta a este problema que nos fuera planteado inicialmente por el antropólogo físico J. Munizaga. Es por esto que hemos considerado importante iniciar estudios conducentes a cubrir este vacío.

No es el objetivo de este trabajo resolver el problema en forma definitiva, sino que más bien abrir una vía de investigación y proponer una manera de solucionarlo en forma relativa quedando para futuro su contrastación en el vivo y ser complementado con otros elementos del esqueleto que contribuyan a dar mayor confiabilidad a las medidas y relaciones aquí sugeridas.

\section{Material}

En nuestro trabajo hemos considerado el uso de costillas como elemento anatómico "duro" de referencia, para estimar la capacidad torácica en forma relativa e inferir indirectamente una aproximación a la capacidad vital.

Para este estudio preliminar hemos empleado terceras y séptimas costillas de tres individuos de sexo femenino y de siete de sexo masculino, provenientes de una colección de restos óseos humanos de la población actual de Santiago, recopilada por J. Munizaga y depositada en el laboratorio de antropología física del Departamento de Antropología de la Universidad de Chile. También se midieron los fémures de los mismos individuos para establecer relaciones entre la estatura y los valores obtenidos para el tórax de cada uno. Se agregaron a esta pequeña muestra tomada al azar datos provenientes de un individuo de sexo femenino del cementerio precolombino de la localidad Caleta Huelén, restos óseos facilitados también por el profesor J. Munizaga.

\section{Método propuesto}

Durante el desarrollo de la presente investigación se procedió a tomar una serie de medidas en el material estudiado, de las cuales sólo se empleó una parte, sin embargo sugerimos tomar aquellas medidas que abajo se indican para explorar otras formas de resolver el problema planteado de una manera diferente a la que aquí se propone.

A cada medida se le asignó arbitrariamente una letra minúscula (de: a, a g), secuencia que se dio a medida que la investigación se fue desarrollando.

a) Distancia, en línea recta, entre la cresta de la cabeza ósea y la parte media, del lado externo del ángulo de la costilla. Esta medida se realiza con compás.

b) Perímetro externo de la costilla. Se mide con una huincha métrica, de género, desde la cresta de la cabeza ósea hasta la parte media de la extremidad anterior.

c) Diámetro entre la cresta de la cabeza ósea y la parte media de la extremidad anterior de la costilla.

\footnotetext{
1 Departamento de Antropología de la Universidad de Chile. Casilla 10136, Correo Central, Santiago, CHILE.
} 
d) Diámetro medido desde la parte media de la extremidad anterior hasta la parte media anterior del ángulo de la costilla

e) Diámetro máximo establecido por una línea perpendicular al diámetro c, partiendo de éste hasta la parte más externa (en la parte media) del cuerpo de la costilla.

f) Alto máximo de la costilla medido a no más de cinco centímetros del ángulo, hacia el cuerpo de esta.

g) Espesor de la costilla medido por delante de la tuberocidad.

Todas las medidas arriba indicadas están representadas en la parte media de la lámina adjunta a este trabajo.

Después de probar una serie de relaciones entre las medidas arriba señaladas para varias costillas y en relación con la estatura, expresada en la longitud de fémur de varios individuos y al no obtener resultados claramente satisfactorios, nos inclinamos por considerar al tórax como un cuerpo geométrico sencillo. Tal como lo señalan algunos autores de conocidos textos de fisiología humana: "El tórax en conjunto adopta la forma de un cono truncado, más ancho en su base, cuyo eje transversal es mayor que el anteroposterior" (Hussay et al. 1952).

El tratar al tórax como a un cono truncado sin duda facilita la resolución del problema antes planteado, pero no está exento de ciertos inconvenientes de orden metodológico-prácticos. Debemos tomar en cuenta que el tórax, si bien asemeja el cuerpo geométrico descrito, posee una serie de irregularidades y asimetrías que debemos compensar de alguna forma. Además la base de este supuesto cono es diferente si consideramos a las últimas costillas como parte de ésta a si consideramos la posición relativa del diafragma en un momento dado. Debemos considerar junto a esto que este supuesto cono modifica sus volúmenes como consecuencia de la mecánica respiratoria.

Si analizamos la fórmula para calcular el volumen de un cono truncado, que se expone en la parte superior de la lámina adjunta a este trabajo, los inconvenientes arriba señalados se hacen evidentes, así como si el elemento anatómico a emplear son algunas costillas se ponen de manifiesto otras dificultades. No tenemos una forma directa de conocer la altura del cono, tampoco las costillas por sí solas forman la totalidad del cono, por lo tanto, aunque éstas formaran un semicírculo, no podemos conocer en forma directa los radios de la base y de la cima. Todo esto hace necesario establecer una serie de consideraciones previas que validen algunas modificaciones que hemos introducido a la fórmula y que justifiquen su aplicabilidad.

Estableceremos las siguientes premisas:

- Las costillas, al ser elementos estructurales permanentes del tórax, deben, de alguna manera, reflejar la forma y tamaño relativo de este, por lo tanto ciertas medidas que den cuenta del tamaño y forma de las costillas lo hacen también indirectamente en relación al tórax. Es posible por ende prescindir otros elementos anatómicos como esternón, cartílagos y vértebras dorsales.

- Si bien la base y la cima del supuesto cono no forman un mismo plano con las respectivas costillas en su posición anatómica normal, es perfectamente posible considerarlas equivalentes al transformarse en mediciones sistemáticas e incluyendo las desviaciones en una constante de corrección. Además aunque una costilla de por sí no trace en su trayectoria el perímetro de un solo plano se puede considerar como formando uno al promediar algunas medidas que reflejen su curvatura.

- Mediante medidas de dos costillas, una de ellas en relación a la base y otra relación a la cima, es posible reconstruir el resto del cono formado por el tórax.

- Podemos establecer una altura para el cono torácico, en forma indirecta del siguiente modo: promediando los valores obtenidos para el alto de las costillas (aquellas consideradas para la reconstrucción de este) y multiplicándolo por un factor sencillo que aproxime esta altura a un valor real de incluirse todas las costillas que forman el cono y los espacios entre ellas.

A las consideraciones arriba señaladas debemos agregar que: al construir un cono que refleje la 
capacidad torácica de un individuo, podemos escoger algunas costillas que muestren mejor esa medida en relación a una situación dinámica y a la adaptación del individuo (en cuanto a su morfología se refiere), a una determinada altura o más bien a una determinada tensión de oxígeno. Para la elección de las citadas costillas debemos señalar, en primer lugar, que las medidas que se tomen tan solo podrán reflejar una situación estática dentro de la mecánica respiratoria de un individuo. No será posible, al menos por ahora y con los elementos que aquí se consideran, dar cuenta de situaciones dinámicas, como valores obtenidos en el vivo al establecer su capacidad vital; aunque en condiciones normales (ausencia de patologías, individuos adultos y otras) sea posible inferir una proporcionalidad entre capacidad vital y volumen torácico. Sin embargo, podemos considerar al tórax relativamente estático durante la respiración tranquila, dado que el movimiento de las costillas es mínimo, siendo el diafragma el que realiza los movimientos que permitan la respiración del individuo (Houssay et al. 1952). En condiciones normales, la respiración tranquila puede ser un buen índice de la adaptación de un individuo a la altura y en ésta jugarán un papel determinante el volumen torácico y, en especial, la superficie formada por la base de este cono truncado y cubierta por el diafragma.

El método aquí propuesto consiste básicamente en reemplazar algunos términos de la fórmula para determinar el volumen de un cono truncado (expuesta en la parte superior de la lámina adjunta) por algunas medidas o promedios de medidas obtenidas en terceras y séptimas costillas, agregándose a la forma una constante de corrección (denominada K), la cual no es determinada en este trabajo pues se requiere realizar mediciones en individuos vivos.

En la parte inferior de la lámina adjunta a este trabajo presentamos la fórmula modificada por nosotros. En ella se puede apreciar que los diferentes términos de la fórmula original han sido reemplazados por medidas o promedios de medidas de terceras y séptimas costillas (indicándose su proveniencia con un subíndice de 3 o 7 respectivamente). Las medidas aparecen designadas por las mismas letras que las usadas para describirlas y que aparecen esquematizadas en la parte central de la lámina.

En primer término de la fórmula corresponde a lo que hemos denominado "índice de capacidad torácica relativa" (iCtr), es decir, el volumen torácico expresado como el de un cono truncado. Este índice está expresado como proporcional $(\alpha)$ al valor obtenido del desarrollo del resto de la fórmula y no como una igualdad para no suscitar equívocos en relación con los valores reales de capacidad torácica que se pudieran obtener en el vivo.

El siguiente término modificado corresponde al valor de la altura del cono truncado (h), que ha sido reemplazada por el valor promedio obtenido para la medida $f$ (ver parte central de la lámina) entre las costillas tercera y séptima de un individuo, el cual es multiplicado por un factor de corrección que hemos estimado en nueve tentativamente, $\left(\mathrm{h}=\left(9 \mathrm{xf}_{3-7}\right)\right)$.

Las expresiones del resto de la fórmula, con excepción de $\mathrm{K}$, corresponden a valores promedios entre los valores obtenidos de las medidas "c" (se considera sólo c/2) y "e", empleándose los valores de una tercera costilla $\left(\mathrm{c}_{3} / 2\right.$ y $\left.\mathrm{e}_{3}\right)$ para determinar el radio uno $\left(\mathrm{r}_{1}\right)$, y los valores obtenidos de una séptima costilla $\left(\mathrm{c}_{7} / 2 \mathrm{y} \mathrm{e}_{7}\right)$ para el radio dos $\left(\mathrm{r}_{2}\right)$, es decir, la base del cono truncado establecido en el tórax de un individuo.

K corresponde a un factor de corrección de la fórmula que deberá ser determinado más tarde en estudios realizados en vivos.

Se estimó conveniente relacionar de alguna manera los valores obtenidos con la talla de los individuos, dada su relación con la necesidad de ventilación de estos y por ende en cómo el tórax satisface esas necesidades en relación a diferentes disponibilidades de oxígeno. Con esta finalidad calculó un índice de capacidad torácica relativa obtenido para un individuo por la longitud, expresada en $\mathrm{cm}$, de uno de sus fémures.

Cabe agregar que todas las mediciones fueron realizadas en centímetros, hasta con un decimal. Todas las operaciones fueron realizadas considerando más de cuatro decimales.

\section{Resultados}

Los resultados obtenidos para nuestra primera muestra se indican en los cuadros 1 y 2 . 


\begin{tabular}{|l|l|l|c|}
\hline \multicolumn{1}{|c|}{ Clave } & Sexo & iCtr & iCtr/fe \\
\hline Pab. 10 ad. N-91 & Femenino & 2041.9445 & 51.0475 \\
\hline G-18 ad. N-466 & Femenino & 2210.1272 & 50.8068 \\
\hline PP N-17 & Femenino & 2156.2666 & 52.8504 \\
\hline C Hue. & Femenino & 2213.2912 & 59.0213 \\
\hline Promedio Cementerio General & & 2136.1128 & \\
\hline Promedio Sexo & & 2155.4049 & 53.2855 \\
\hline
\end{tabular}

Cuadro 1. Valores obtenidos para los individuos femeninos.

\begin{tabular}{|c|c|c|c|}
\hline Clave & Sexo & iCtr & iCtr/fe \\
\hline Gal 17 ad. N-216 & Masculino & 3812.7541 & 100.335 \\
\hline Gal 18 ad. N-86 & Masculino & 2238.7510 & 48.88 \\
\hline P-36 S-1333 & Masculino & 2671.3884 & 67.4595 \\
\hline Pab. B ad. N-51 & Masculino & 3560.8978 & 75.124 \\
\hline P-36 S-1116 & Masculino & 3083.9204 & 69.301 \\
\hline P-6 S-412 & Masculino & 2711.0126 & 65.32 \\
\hline Gal 18 ad. N-646 & Masculino & 3597.2648 & 77.695 \\
\hline Promedio Cementerio General & & 3096.5699 & 72.016 \\
\hline
\end{tabular}

Cuadro 2. Valores obtenidos para los individuos masculinos.

\section{Discusión}

A pesar de lo reducido de esta nuestra inicial es posible diferenciar las capacidades torácicas relativas de las mujeres de las de los hombres, diferencia originada básicamente en el dimorfismo sexual. No es necesario hacer uso de ningún recurso estadístico para mostrar el agrupamiento diferencial de cifras por sexo, aunque en el futuro nos resulte necesario, eventualmente, para discriminar a individuos de poblaciones diferentes como las del altiplano que por el momento no disponemos.

Se puede apreciar además, aunque esto pueda ser casual y estadísticamente no significativo, que existe una sugerente diferencia entre los valores obtenidos para el iCtr/fe de las mujeres de la población de Santiago y la mujer de Caleta Huelén. Esto podría estar indicando que el método propuesto podría discriminar bien entre diferentes poblaciones pero es necesario todavía verificarlo con una muestra mucho más amplia, quedando por ahora esto como una observación tentativa.

En relación a la fórmula misma, su aplicación nos ha mostrado que ésta podría estar reflejando adecuadamente las características para las cuales fue construida, en especial si se comparan los datos, guardando las debidas precauciones (y proporciones), con valores relacionados con la capacidad torácica como es el caso de la capacidad vital en poblaciones vivas (Vital and Health Statistics. Series 11, no 22 - 1971-75). Esto es, siempre que se emplee un criterio poblacional y se trabaje en condiciones normales.

Con respecto al valor de $h$, hemos preferido estimarlo provisoriamente en 9 para no distorsionar el sentido real que hemos querido darle a la fórmula -el de una cierta proporcionalidad con valores reales de capacidad torácica- no asignándole un valor que arroje por el momento valores similares a medidas obtenidas en el vivo; este sería el caso si se usaran cifras próximas a 13.

$\mathrm{Al}$ relacionar los valores obtenidos para el iCtr con la longitud del fémur, se lo ha hecho pensando en individuos normales dentro de una población; de cumplirse lo anterior los resultados deberían presentarse sin distorsiones.

\section{Conclusiones}

Creemos que la fórmula desarrollada por nosotros puede, con las debidas salvedades, arrojar resultados 


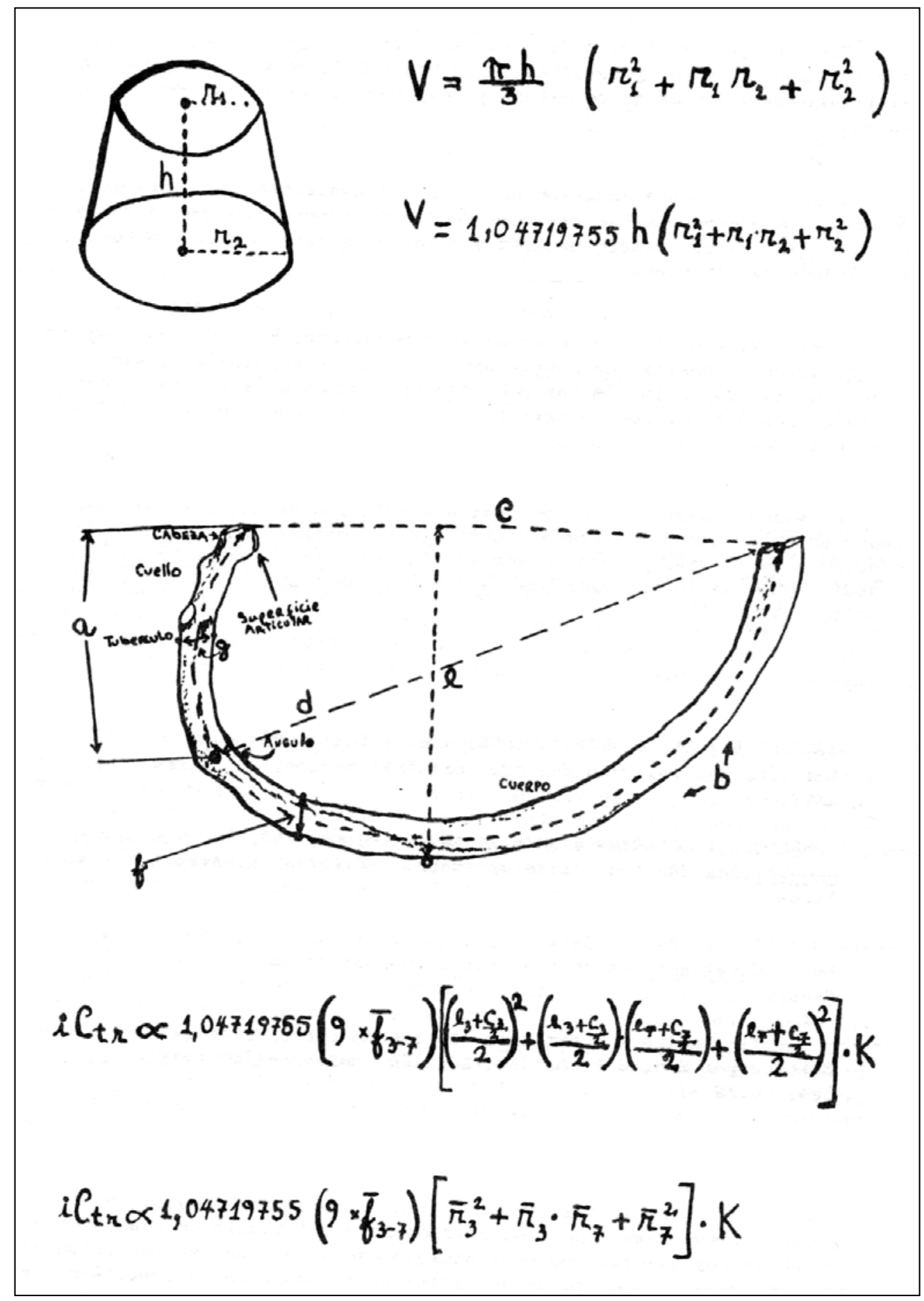

Figura 1. Indice de capacidad torácica relativa. 
que hagan útil su aplicación al estudiar poblaciones indígenas de altura y compararlas con otras de zonas más bajas.

Los valores hasta ahora obtenidos en el desarrollo de la investigación muestran que es posible reflejar de alguna forma la capacidad torácica de un individuo usando medidas y relaciones entre éstas obtenidas en algunos de sus huesos.

Esta investigación, si bien no es concluyente, muestra un campo de investigación necesario de cubrir dentro de la antropología física y el estudio de la adaptación de las poblaciones humanas a la altura, insinuando tanto las dificultades existentes como las grandes posibilidades futuras en el desarrollo del problema.

No siendo nuestro objetivo dar una solución definitiva al tema planteado en este trabajo, creemos contribuir al mostrar una posible vía de solución a éste susceptible de ser modificada o reemplazada a futuro en la medida que las investigaciones se desarrollen o que los datos biológicos lo posibiliten.

\section{REFERENCIAS CITADAS}

HOUSSAY, B. A., J. LEWIS y otros, 1952. Fisiología humana, $2^{a}$ Edición. Editorial El Ateneo, Buenos Aires.

HOUSSAY, B. A., R. CALDEYRO-BARCIA y otros, 1972. Fisiología humana, $4^{\text {a }}$ Edición. Editorial El Ateneo, Buenos Aires.
— 1971-75. Basic. Data on spirometry in adults 25-74 years of age. Vital and Health Statistics Series 11 (22).

1971. Tablas científicas. Ciba Geigy S.A., Basilea. 